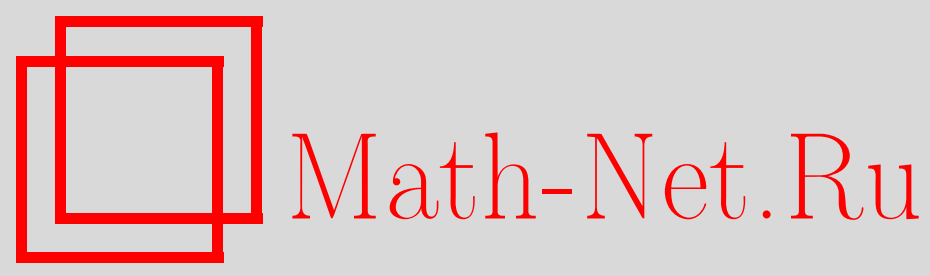

В. Ю. Попов, Многообразие колец, не обладающее независимым базисом, Матем. заметки, 2001, том 69, выпуск $5,713-732$

DOI: https://doi.org/10.4213/mzm535

Использование Общероссийского математического портала Math-Net.Ru подразумевает, что вы прочитали и согласны с пользовательским соглашением http://www . mathnet.ru/rus/agreement

Параметры загрузки:

IP: 3.80 .253 .173

26 апреля 2023 г., 03:58:02

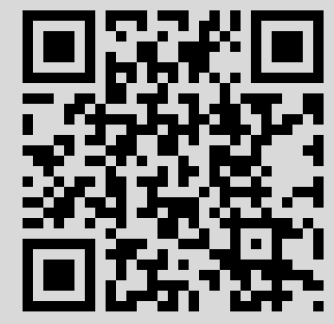




\section{МНОГООБРАЗИЕ КОЛЕЦ,}

\section{НЕ ОБЛАДАЮЩЕЕ НЕЗАВИСИМЫМ БАЗИСОМ}

\section{В. Ю. Попов}

Построен пример многообразия колец, не обладающего независимьм базисом. Доказано, что это многообразие является пересечением двух независимо базируемых многообразий.

Библиография: 10 названий.

Система тождеств $\left\{u_{i}=v_{i} \mid i \in I\right\}$ называется независимой, если никакое из тождеств этой системы не является следствием всех остальных (см., например, $[1$, с. 148]). Говорят, что многообразие алгебрачческих систем независимо базируемо, если существует независимая система тождеств, являющаяся базисом этого многообразия.

Любое многообразие, которое может быть определено конечной системой тождеств, очевидно, может быть задано и независимой системой тождеств. В работах [2] и [3] доказано, что каждое многообразие полугрупп, заданное уравновешенными и 0-приведенными тождествами, обладает независимым базисом. Первый пример многообразия полугрупп, не имеющего независимого базиса, построен в работе [4]. Другие примеры многообразий полугрупा, не обладаюших независимым базисом, построены в работах [5]-[7]. В [5] приведен пример системы тождеств моноидов, в [8] - инверсных полугруп, в [9] - груп, не имеюших независимого базиса. В [6] доказано, что существует многообразие полугруп, порожденное конечной полугруппой и не имеющее независимого базиса. Заметим, что любое полиоднородное многообразие колец, а значит и многообразие алгебр над бесконечным полем, независимо базируемо, поскольку тождества меньшей степени не следуют из тождеств большей степени. Цель настоящей заметки построение многообразия колец, не имеющего независимого базиса.

Теорема. Существует многообразие колеи, X, не обладающее независимым базисом.

Введем ряд обозначений. Пусть $\mathscr{K}$ - некоторое, не обязательно ассоциативное кольцо, $a_{1}, a_{2}, \ldots, a_{n-1}, a_{n} \in \mathscr{K}$. Определим левонормированное произведение $a_{1} a_{2} \ldots a_{n-1} a_{n}$ индукцией по $n$, полагая $a_{1} a_{2} \ldots a_{n-1} a_{n}=\left(a_{1} a_{2} \ldots a_{n-1}\right) a_{n}$ при $n>1$. При $n=1$ имеем просто $a_{1}$. Более того, полагаем

$$
a_{1} \underbrace{a_{2} \ldots a_{2}}_{m \text { pa3 }}=a_{1} a_{2}^{m},
$$

$b^{0}$ - пустой символ для любого символа $b$. Пусть $X=\left\{x_{1}, x_{2}, \ldots, x_{n}, \ldots\right\}$-некоторое счетное множество, $\Gamma$ - свободный группоид с множеством свободных образуюших $X$. 
Обозначим через $F$ свободное кольцо, базисом которого как $\mathbb{Z}$-модуля является множество Г. Определим линейные отображения $A, B, C, D$, переводящие элементы декартова произведения $F \times F$ в элементы кольца $F$, полагая

$$
\begin{array}{ll}
A(y, x)=\left(x\left(x(y x) x^{2}\right) x\right), & C(y, x)=\left(x\left(x(y x) x^{4}\right) x\right) \\
B(y, x)=\left(x\left(x(y x) x^{3}\right) x\right), & D(y, x)=\left(x\left(x(y x) x^{5}\right) x\right)
\end{array}
$$

Если $Y_{1}=Y_{1}(x, y), Y_{2}=Y_{2}(x, y), \ldots, Y_{n-1}=Y_{n-1}(x, y), Y_{n}=Y_{n}(x, y)$ - некоторые отображения, то суперпозишию этих отображений $Y_{2}\left(Y_{1}\left(z_{1}, z_{2}\right), z_{3}\right)$ будем обозначать через $Y_{1}\left(z_{1}, z_{2}\right) Y_{2}\left(z_{3}\right)$; распространяя это обозначение индукцией по $n$, полагаем

$$
Y_{n}\left(Y_{1}\left(z_{1}, z_{2}\right) Y_{2}\left(z_{3}\right) \ldots Y_{n-1}\left(z_{n}\right), z_{n+1}\right)=Y_{1}\left(z_{1}, z_{2}\right) Y_{2}\left(z_{3}\right) \ldots Y_{n-1}\left(z_{n}\right) Y_{n}\left(z_{n+1}\right)
$$

Кроме того, считаем, что

$$
X\left(z_{1}, z_{2}\right) Y^{n}\left(z_{3}\right)=X\left(z_{1}, z_{2}\right) \underbrace{Y\left(z_{3}\right) \ldots Y\left(z_{3}\right)}_{n \text { раз }} .
$$

Пусть $\Re$ - многообразие всех метабелевых колец характеристики $2 ; F \Re-$ кольцо, свободное в многообразии $\mathfrak{R}$ и имеющее счетное множество свободных образующих $\left\{e, e_{1}\right.$, $\left.\ldots, e_{n}, \ldots\right\}, F_{1} \mathfrak{R}-$ моногенное кольцо, свободное в многообразии $\mathfrak{R}$. Пусть $\mathfrak{Y}$ - многообразие, заданное тождеством

$$
B(z, x) B(x) C(x) D(x)=B(z, x) C(x)
$$

и системой тождеств

$$
A(x, x) B^{n}(x) C(x)=0,
$$

где $n \in \mathbb{N} ; \mathfrak{Y}_{m}-$ многообразие, заданное тождеством (1) и системой тождеств вида (2), где $n<m$. Положим $\mathfrak{X}=\mathfrak{Y} \cap \mathfrak{R}$. Обозначим через $\mathfrak{X}_{m}, F \mathfrak{X}, F \mathfrak{X}_{m}$ многообразие $\mathfrak{Y}_{m} \cap \mathfrak{R}$; кольцо счетного ранга, свободное в многообразии $\mathfrak{X}$; кольцо счетного ранга, свободное в многообразии $\mathfrak{X}_{m}$, соответственно. Для удобства образующие колец $F \mathfrak{X}$ и $F \mathfrak{X}_{m}$ будем обозначать теми же буквами, что и образующие кольца $F \Re$.

ДоКАЗАТЕЛЬСТВО ТЕОРЕМЫ. Покажем, что многообразие $\mathfrak{X}$ не имеет независимого базиса. Для этого нам потребуются следующие две леммы.

Лемма 1. Для любого $n>1$ из тождеств (1) и (2) следует тождество

$$
A(x, x) B^{m}(x) C(x)=0,
$$

əде $m \in\{1, \ldots, n-1\}$. 
ДоКАЗАТЕЛЬСТВО проведем индукцией по $n$.

База индукции. Пусть $n=2$. Тогда нам нужно показать, что из выполнимости тождеств (1) и

$$
A(x, x) B^{2}(x) C(x)=0
$$

следует тождество $A(x, x) B(x) C(x)=0$. В самом деле, из выполнимости тождества (4) вытекает вьполнимость тождества $A(x, x) B^{2}(x) C(x) D(x)=0$. Отсюда в силу тождества (1) получаем

$$
\begin{aligned}
A(x, x) B^{2}(x) C(x) D(x) & =B(A(x, x), x) B(x) C(x) D(x) \\
& =B(A(x, x), x) C(x)=A(x, x) B(x) C(x)=0,
\end{aligned}
$$

что и требовалось доказать.

Шаг индукции. Допустим, что утверждение леммы справедливо для некоторого $n$. Покажем, что оно вьполняется и для $n+1$. Покажем сначала, что из тождеств $(1)$ и

$$
A(x, x) B^{n+1}(x) C(x)=0
$$

следует тождество (2). В самом деле, из выполнимости тождества (5) вытекает выполнимость тождества $A(x, x) B^{n+1}(x) C(x) D(x)=0$. Отсюда в силу тождества (1) получаем следующую цепочку тождеств:

$$
\begin{aligned}
A(x, x) B^{n+1}(x) C(x) D(x) & =B\left(A(x, x) B^{n-1}(x), x\right) B(x) C(x) D(x) \\
& =B\left(A(x, x) B^{n-1}(x), x\right) C(x)=A(x, x) B^{n}(x) C(x)=0
\end{aligned}
$$

что и требовалось доказать. По предположению индукции из тождеств (1) и (2) следует тождество (3), где $m \in\{1, \ldots, n-1\}$. С учетом того, что из тождеств (1) и (5) следует тождество (2), получаем, что из тождеств (1) и (5) следует тождество (3), где $m \in\{1, \ldots, n\}$. Лемма 1 доказана.

ЛЕмма 2. Для любого натурального числа $n$ тождество (2) выполняется в многообразии $\mathfrak{X}_{m}$ тогда и только тогда, когда $n<m$.

ДокАЗАТЕЛЬСтво. Допустим, что $n<m$. Тогда тождество (2) вьполняется в многообразии $\mathfrak{X}_{m}$ по определению многообразия $\mathfrak{X}_{m}$. Допустим теперь, что $n \geqslant m$. Нам нужно показать, что тождество $(2)$ не вьполняется в многообразии $\mathfrak{X}_{m}$. Предположим противное. Из выполнимости тождества (2) в многообразии $\mathfrak{X}_{m}$ вытекает выполнимость этого тождества в кольце $F \mathfrak{X}_{m}$. Тождество $(2)$ выполняется в кольце $F \mathfrak{X}_{m}$ тогда и только тогда, когда в кольце $F \mathfrak{X}_{m}$ вьполняется равенство

$$
A(e, e) B^{n}(e) C(e)=0 .
$$

Обозначим через $S$ множество многочленов $f(e)$ таких, что $f(e)=0$ - соотношение в кольце $F \mathfrak{X}_{m}$, полученное подстановкой конкретных элементов кольца $F \mathfrak{X}_{m}$ вместо переменных в тождество (1) или в тождество

$$
A(x, x) B^{m-1}(x) C(x)=0 .
$$


Пусть $I$ - идеал кольца $F \Re$, порожденный множеством $S$. Согласно лемме 1 имеет место равенство

$$
\mathfrak{X}_{m}=\operatorname{var}\left\{B(z, x) B(x) C(x) D(x)=B(z, x) C(x), A(x, x) B^{m-1}(x) C(x)=0\right\} .
$$

Следовательно, равенство (6) вьполняется в кольце $F \mathfrak{X}_{m}$ тогда и только тогда, когда многочлен

$$
A(e, e) B^{n}(e) C(e)
$$

принадлежит идеалу $I$. Пусть

$$
u_{i 1} h_{j} u_{i 2}
$$

- некоторое произведение многочленов, имеющее вид $u_{i_{1}}^{(1)} \ldots u_{i_{1}}^{(k)} h_{j} u_{i_{2}}^{(1)} \ldots u_{i_{2}}^{(l)}$ с некоторым расположением скобок. Тогда принадлежность многочлена (8) идеалу I равносильна тому, что в кольце $F \Re$ вьполняется равенство вида

$$
A(e, e) B^{n}(e) C(e)=\sum_{i, j} u_{i 1} h_{j} u_{i 2},
$$

где $h_{j} \in S$. В силу дистрибутивности можно считать, что все многочлены, входящие в $(9)$, за исключением $h_{j}$, являются одночленами. Поскольку многообразие $\mathfrak{R}$, а значит и кольцо $F \Re$, удовлетворяет тождеству $(x y)(z t)=0$, то все эти одночлены являются суммами свободных образующих. Так как в левой части равенства (10) стоит многочлен, зависящий только от $e$, то в силу свободы кольца $F \Re$ можно считать, что многочлен

$$
\sum_{i, j} u_{i 1} h_{j} u_{i 2}
$$

тоже зависит только от $e$. Следовательно, равенство (10) вьполняется в кольце $F \Re$ тогда и только тогда, когда оно вьполняется в кольце $F_{1} \Re$. Для приведения многочлена (11) к более простому виду нам потребуется следующая

Лемма 3. Пусть $W \rightleftharpoons\left\{A(e, e) B^{n}(e) C(e) D^{k}(e) \mid n \in \mathbb{N}, k \in \mathbb{N} \cup\{0\}\right\}$. Тогдa сумма (11) представима в виде $f_{1}+f_{2}$, где $f_{1}$ - многочлен, который сам может быть представлен в виде (11), каждый одночлен многочлена $f_{1}$ принадлежит мнохеству $W$, а $f_{2}$ - многочлен, ни один одночлен которого не принадлежит множеству $W$.

ДоКАЗАТЕЛЬСТВО проведем индукцией по числу одночленов в сумме (11). Допустим, что число $d$ одночленов в сумме (11) равно нулю. Тогда утверждение очевидно. Допустим, что утверждение справедливо для некоторого числа одночленов $d$. Покажем, что оно верно и для $d+1$. Пусть число одночленов в сумме $(11)$ не больше $d+1$. Тогда

$$
\sum_{i, j} u_{i 1} h_{j} u_{i 2}=\sum_{i>1, j} u_{i 1} h_{j} u_{i 2}+u_{11} h_{1} u_{12} .
$$

Так как число одночленов в сумме

$$
\sum_{i>1, j} u_{i 1} h_{j} u_{i 2}
$$


не превосходит $d$, то по предположению индукции имеем, что

$$
\sum_{i>1, j} u_{i 1} h_{j} u_{i 2}=f_{1}+f_{2} .
$$

Рассмотрим многочлен

$$
u_{11} h_{1} u_{12} \text {. }
$$

В зависимости от того, получен ли многочлен $h_{1}$ из тождества (1) или из тождества (7), следует рассмотреть два случая.

Пусть $h_{1}$ получен из тождества $(7)$. В силу метабелевости кольца $F_{1} \Re$ получаем, что либо $h_{1}=0$, либо $h_{1}=A(e, e) B^{m-1}(e) C(e)$, либо

$$
h_{1}=A(e, e) B^{m-1}(e) C(e)+A(y, e) B^{m-1}(e) C(e)+A(e, y) B^{m-1}(e) C(e),
$$

где $y$ - многочлен, отличньй от нуля и не содержаший одночлена $e$. Поскольку многочлен $y$ входит в многочлены

$$
A(y, e) B^{m-1}(e) C(e)
$$

и

$$
A(e, y) B^{m-1}(e) C(e)
$$

линейно, в силу дистрибутивности $y$ можно считать одночленом. Покажем, что имеют место следующие два соотношения:

$$
\begin{aligned}
& u_{11}\left(A(y, e) B^{m-1}(e) C(e)\right) u_{12} \notin W \\
& u_{11}\left(A(e, y) B^{m-1}(e) C(e)\right) u_{12} \notin W .
\end{aligned}
$$

Рассмотрим сначала случай слова $u_{11}\left(A(y, e) B^{m-1}(e) C(e)\right) u_{12}$. Допустим, что слово $u_{11}\left(A(y, e) B^{m-1}(e) C(e)\right) u_{12} \in W$. Тогда для некоторых $n$ и $k$ должно выполняться равенство $A(e, e) B^{n}(e) C(e) D^{k}(e)=u_{11}\left(A(y, e) B^{m-1}(e) C(e)\right) u_{12}$. Следовательно, слово (16) является подсловом слова

$$
A(e, e) B^{n}(e) C(e) D^{k}(e)
$$

Предположим, что

$$
A(e, e) B^{n}(e) C(e) D^{k}(e)=A(y, e) B^{m-1}(e) C(e) .
$$

Следует рассмотреть два случая в зависимости от того, будет ли $k$ числом положительным или $k=0$. Допустим, что $k>0$. Тогда из равенства $(21)$ непосредственно вытекает равенство $\left(e\left(A(e, e) B^{n}(e) C(e) D^{k-1}(e) e\right)\right) e=e\left(A(y, e) B^{m-1}(e) e\right)$. Последнее равенство равносильно в кольце $F_{1} \Re$ следующей системе равенств:

$$
e\left(A(e, e) B^{n}(e) C(e) D^{k-1}(e) e\right)=e, \quad e=A(y, e) B^{m-1}(e) e .
$$

Очевидно, что оба эти равенства в кольце $F_{1} \mathfrak{R}$ ложны. Предположим теперь, что $k=0$. Тогда равенство (21) принимает вид $A(e, e) B^{n}(e) C(e)=A(y, e) B^{m-1}(e) C(e)$. Очевидно, что это равенство равносильно в кольце $F_{1} \Re$ равенству

$$
A(e, e) B^{n}(e)=A(y, e) B^{m-1}(e) .
$$


Предположим, что $n>m-1$. Тогда из равенства (22) следует равенство

$$
A(e, e) B^{n-m+1}(e)=A(y, e) .
$$

Из этого равенства получаем равенство $\left(e\left(A(e, e) B^{n-m}(e) e\right)\right) e=e(A(y, e) e)$, равносильное в кольце $F_{1} \Re$ ложной системе равенств $e\left(A(e, e) B^{n-m}(e) e\right)=e, e=A(y, e) e$. Предположим теперь, что $n<m-1$. Тогда из равенства (22) вытекает равенство $A(e, e)=A(y, e) B^{m-1-n}(e)$, равносильное равенству $e(e e)=\left(e\left(A(y, e) B^{m-2-n}(e) e\right)\right) e$, которое в свою очередь равносильно ложной системе равенств

$$
e e=e, \quad e=e\left(A(y, e) B^{m-2-n}(e) e\right) .
$$

Пусть теперь $n=m-1$. В этом случае, как нетрудно убедиться, из равенства $(22)$ вытекает равенство $e=y$, которое противоречит нашему предположению о том, что $e \neq y$. Таким образом, мы убедились в том, что равенство (21) не вьполняется. Следовательно, слово (16) является собственным подсловом слова (20). Несложная проверка позволяет убедиться в том, что это невозможно. Следовательно, имеет место соотношение (18). Совершенно аналогично можно рассмотреть случай слова (17) и убедиться в выполнимости соотношения (19). Следовательно, требуемьм представлением будет либо представление

$$
\begin{aligned}
& \left(u_{11}\left(A(e, e) B^{m-1}(e) C(e)\right) u_{12}+f_{1}\right) \\
& \quad+\left(u_{11}\left(A(y, e) B^{m-1}(e) C(e)\right) u_{12}+u_{11}\left(A(e, y) B^{m-1}(e) C(e)\right) u_{12}+f_{2}\right)
\end{aligned}
$$

либо представление

$$
\begin{aligned}
f_{1}+ & \left(u_{11}\left(A(e, e) B^{m-1}(e) C(e)\right) u_{12}\right. \\
& \left.+u_{11}\left(A(y, e) B^{m-1}(e) C(e)\right) u_{12}+u_{11}\left(A(e, y) B^{m-1}(e) C(e)\right) u_{12}+f_{2}\right) .
\end{aligned}
$$

Аналогично можно рассмотреть случай, когда многочлен $h_{1}$ получен из тождества (1). Лемма 3 доказана.

Вообще говоря, если в кольце $F_{1} \mathfrak{R}$ вьполняется равенство вида $(10)$, то таких равенств для различных многочленов (11) выполняется бесконечно много. Заметим, что слово (8) принадлежит множеству $W$. Поэтому в силу свободы кольца $F_{1} \Re$ из равенства $A(e, e) B^{n}(e) C(e)=f_{1}+f_{2}$ вытекает равенство $f_{2}=0$. В дальнейшем мы будем рассматривать такое равенство (10), что число одночленов в многочлене (11) минимально. Учитывая лемму 3 , легко понять, что в этом случае будет иметь место равенство $\sum_{i, j} u_{i 1} h_{j} u_{i 2}=f_{1}$. Завершают доказательство леммы 2 индуктивные рассуждения, которые мы оформим в отдельную лемму.

ЛЕмМА 4. Для любого $k \geqslant 0$ и для любого $n>m+k-1$ равенство

$$
A(e, e) B^{n}(e) C(e) D^{k}(e)=\sum_{i, j} u_{i 1} h_{j} u_{i 2}
$$

ложнно в кольие $F_{1} \Re$. 
ДоКАЗАТЕЛЬСТво проведем индукцией по числу $p$ одночленов в сумме (11). Предположим, что $p=0$. Тогда утверждение очевидно. Допустим, что утверждение леммы справедливо для некоторого числа $p$, и покажем, что оно выполняется и для $p+1$.

Пусть в кольце $F_{1} \Re$ выполняется равенство $(25)$ и число одночленов в сумме $(11)$ не превосходит $p+1$. Так как (25)- равенство в свободном кольце, найдутся $i$ и $j$ такие, что одночлен (20) будет одночленом многочлена (9). Без ограничения общности можно считать, что $i=j=1$. Следует рассмотреть два случая в зависимости от того, соответствует многочлен $h_{1}$ тождеству (1) или тождеству (7). Допустим, что многочлен $h_{1}$ соответствует тождеству $(1)$. Тогда в кольце $F_{1} \mathfrak{R}$ для некоторого неассоциативного слова $z$ выполняется одно из следующих двух равенств:

$$
\begin{aligned}
& A(e, e) B^{n}(e) C(e) D^{k}(e)=u_{11} B(z, e) B(e) C(e) D(e) u_{12}, \\
& A(e, e) B^{n}(e) C(e) D^{k}(e)=u_{11} B(z, e) C(e) u_{12} .
\end{aligned}
$$

Допустим, что имеет место равенство (26). Несложно убедиться в том, что слово $u_{11} B(z, e) B(e) C(e) D(e) u_{12}$ может быть представлено в виде

$$
u_{11} D(B(z, e) B(e) C(e), e) u_{12}
$$

В силу того, что (26) - равенство в свободном кольце, слово (20) должно содержать подслово

$$
D(B(z, e) B(e) C(e), e) .
$$

Следует рассмотреть два случая в зависимости от того, будет ли равно нулю число $k$. Допустим, что $k=0$. Тогда слово (20) примет вид (8), а равенство (26) примет вид

$$
A(e, e) B^{n}(e) C(e)=u_{11} B(z, e) B(e) C(e) D(e) u_{12}
$$

Предположим, что в кольце $F_{1} \Re$ выполняется равенство

$$
A(e, e) B^{n}(e) C(e)=D(B(z, e) B(e) C(e), e) .
$$

Тогда в силу свободы кольца $F_{1} \Re$ из этого равенства непосредственно вытекает равенство

$$
e\left(A(e, e) B^{n}(e) e\right)=(e(B(z, e) B(e) C(e) e)) e .
$$

Последнее равенство равносильно в кольце $F_{1} \Re$ следующей системе равенств:

$$
e=e(B(z, e) B(e) C(e) e), \quad A(e, e) B^{n}(e) e=e .
$$

Очевидно, что оба эти равенства ложны. Следовательно, ложно и равенство (30). Отсюда непосредственно вытекает, что слово (28) является собственным подсловом слова (8). Заметим, что слово (28) либо является подсловом слова $A(e, e)$, либо равно одному из следующих девяти подслов:

$$
\begin{gathered}
e\left(\left(\left(\left(\left(e\left(A(e, e) B^{n}(e) e\right)\right) e\right) e\right) e\right) e\right) \\
\left(e\left(A(e, e) B^{n}(e) e\right)\right) e^{r}, \quad r \in\{1,2,3,4\}, \\
e\left(A(e, e) B^{p}(e) e\right), \quad p \leqslant n
\end{gathered}
$$




$$
\begin{gathered}
A(e, e) B^{p}(e) e, \quad p \leqslant n, \\
A(e, e) B^{p}(e), \quad p \leqslant n, \\
e\left(\left(\left(\left(e\left(A(e, e) B^{p}(e) e\right)\right) e\right) e\right) e\right), \quad p<n, \\
\left(e\left(A(e, e) B^{p}(e) e\right)\right) e^{r}, \quad p<n, \quad r \in\{1,2,3\}, \\
e\left(A(e, e) B^{p}(e) e\right), \quad p<n, \\
A(e, e) B^{p}(e) e, \quad p<n .
\end{gathered}
$$

Заметим, что длина слова $A(e, e)$ равна 7 , а длина слова (28) больше 34 . Следовательно, слово (28) не является подсловом слова $A(e, e)$. Допустим, что слово (28) равно слову (31). Тогда в кольце $F_{1} \Re$ должно вьполняться равенство

$$
\left(\left(\left(\left(e\left(A(e, e) B^{n}(e) e\right)\right) e\right) e\right) e\right) e=e
$$

что невозможно. Если слово (28) равно слову (32), то при $r \geqslant 3$ в кольце $F_{1} \Re$ должно выполняться ложное равенство $\left(e\left(A(e, e) B^{n}(e) e\right)\right) e^{r-2}=e$. Допустим, что $r<3$ и слово (28) равно слову (32). Если $r=2$, то из равенства

$$
\left(\left(e\left(A(e, e) B^{n}(e) e\right)\right) e\right) e=D(B(z, e) B(e) C(e), e)
$$

очевидньм образом вытекает равенство

$$
\left.\left(e\left(A(e, e) B^{n}(e) e\right)\right) e=e(((((e(B(z, e) B(e) C(e) e)) e) e) e) e) e\right)
$$

Последнее равенство равносильно в кольце $F_{1} \Re$ следующей системе равенств:

$$
\left.e\left(A(e, e) B^{n}(e) e\right)=e, \quad e=((((e(B(z, e) B(e) C(e) e)) e) e) e) e\right) e .
$$

Очевидно, что оба равенства этой системы ложны в кольце $F_{1} \Re$. Если $r=1$, то из равенства

$$
\left(e\left(A(e, e) B^{n}(e) e\right)\right) e=D(B(z, e) B(e) C(e), e)
$$

очевидньм образом вытекает равенство

$$
e\left(\left(\left(\left(e\left(A(e, e) B^{n-1}(e) e\right)\right) e\right) e\right) e\right)=(((e(B(z, e) B(e) C(e) e)) e) e) e
$$

которое равносильно в кольце $F_{1} \mathfrak{R}$ системе ложных равенств

$$
e=((e(B(z, e) B(e) C(e) e)) e) e, \quad\left(\left(\left(e\left(A(e, e) B^{n-1}(e) e\right)\right) e\right) e\right) e=e
$$

Итак, мы убедились, что слово (28) не равно слову (32). Случаи (33)-(39) рассматриваются совершенно аналогично. Следовательно, слово (28) не является подсловом слова (8). Поэтому в кольце $F_{1} \mathfrak{R}$ не выполняется равенство (29). Рассмотрим теперь случай $k>0$. Так же, как и в случае $k=0$, предположим сначала, что

$$
A(e, e) B^{n}(e) C(e) D^{k}(e)=B(z, e) B(e) C(e) D(e) .
$$


Пусть $k=1$. Несложной проверкой можно убедиться в том, что это равенство выполняется тогда и только тогда, когда $A(e, e) B^{n-2}(e)=z$. Отсюда в силу леммы 3 непосредственно вытекает, что имеет место равенство

$$
A(e, e) B^{n-1}(e) C(e) D^{k-1}(e)=\sum_{i>1, j} u_{i 1} h_{j} u_{i 2} .
$$

Заметим, что это равенство удовлетворяет условиям леммы и число одночленов в сумме (13) не превосходит $p$. Это противоречит предположению индукции. Пусть теперь $k>1$. Тогда из равенства (40) вытекает равенство

$$
A(e, e) B^{n}(e) C(e) D^{k-1}(e)=B(z, e) B(e) C(e),
$$

которое в свою очередь равносильно равенству

$$
\left(e\left(A(e, e) B^{n}(e) C(e) D^{k-2}(e) e\right)\right) e=e(B(z, e) B(e) e) .
$$

Очевидно, что последнее равенство равносильно ложной системе равенств

$$
e\left(A(e, e) B^{n}(e) C(e) D^{k-2}(e) e\right)=e, \quad e=B(z, e) B(e) e .
$$

Следовательно, равенство (40) не имеет места. Таким образом, нам осталось рассмотреть случай, когда (28) - собственное подслово слова (20). Заметим, что в этом случае слово (28) либо является подсловом слова (8), либо равно одному из следующих четырех подслов:

$$
\begin{gathered}
\left.e\left(\left(\left(\left(\left(e\left(A(e, e) B^{n}(e) C(e) D^{q}(e) e\right)\right) e\right) e\right) e\right) e\right) e\right) \\
\left(e\left(A(e, e) B^{n}(e) C(e) D^{q}(e) e\right)\right) e^{r}, \quad r \in\{1, \ldots, 5\} \\
e\left(A(e, e) B^{n}(e) C(e) D^{q}(e) e\right) \\
A(e, e) B^{n}(e) C(e) D^{q}(e) e .
\end{gathered}
$$

Случай, когда слово (28) является подсловом слова (8), рассмотрен нами выше. Несложная проверка, позволяет убедиться в том, что слово (28) не равно ни одному из слов (41)-(44). Итак, мы завершили рассмотрение равенства (26). Допустим теперь, что в кольце $F_{1} \mathfrak{R}$ имеет место равенство (27). Рассмотрим сначала случай $k=0$. В этом случае равенство (27) примет вид

$$
A(e, e) B^{n}(e) C(e)=u_{11} B(z, e) C(e) u_{12} .
$$

Предположим, что слово

$$
B(z, e) C(e)
$$

является собственным подсловом слова

$$
u_{11} B(z, e) C(e) u_{12} \text {. }
$$

Тогда в силу равенства (45) слово (46) является собственным подсловом слова (8). В этом случае, как замечено выше, слово (46) либо является подсловом слова $A(e, e)$, 
либо равно одному из слов (31)-(39). Поскольку длина слова (46) больше длины слова $A(e, e)$, слово (46) не может быть подсловом слова $A(e, e)$. Следовательно, слово (46) должно быть равно одному из слов (31)-(39). Однако, как показьвает несложная проверка, это невозможно. Отсюда непосредственно получаем, что слова (46) и (47) равны. Следовательно, равны слова (8) и (46). Легко проверить, что отсюда вытекает равенство

$$
A(e, e) B^{n-1}(e)=z \text {. }
$$

Заметим, что в рассматриваемом случае многочлен $h_{1}$ соответствует тождеству (1). Следовательно, имеет место равенство

$$
u_{11} h_{1} u_{12}=u_{11}(B(z, e) B(e) C(e) D(e)-B(z, e) C(e)) u_{12} .
$$

Поэтому из равенства (48) вытекает равенство

$$
A(e, e) B^{n+1}(e) C(e) D(e)=\sum_{i>1, j} u_{i 1} h_{j} u_{i 2} .
$$

Заметим, что это равенство удовлетворяет условиям леммы и число одночленов в сумме (13) не превосходит $p$. Следовательно, по предположению индукции это равенство ложно. Рассмотрим теперь случай $k>0$. Предположим, что слово (46) является собственньг подсловом слова (47). Тогда в силу равенства (27) слово (46) является собственньп подсловом слова (20). В этом случае слово (46) либо является собственньпм подсловом слова (8), либо равно одному из слов (41)-(44). Случай, когда слово (46) является подсловом слова (8), рассмотрен нами вьше. Несложная проверка позволяет убедиться в том, что слово (46) не равно ни одному из слов (41)-(44). Предположим теперь, что имеет место равенство $A(e, e) B^{n}(e) C(e) D^{k}(e)=B(z, e) C(e)$. Из этого равенства очевидньг образом вытекает равенство

$$
\left(e\left(A(e, e) B^{n}(e) C(e) D^{k-1}(e) e\right)\right) e=e(B(z, e) C(e) e)
$$

которое, в свою очередь, равносильно ложной системе равенств

$$
e\left(A(e, e) B^{n}(e) C(e) D^{k-1}(e) e\right)=e, \quad B(z, e) C(e) e=e .
$$

Аналогично тому, как мы рассмотрели тождество (1), можно рассмотреть и случай тождества (7). Лемма 4 доказана.

Из леммы 4 вытекает, что для любого $n>m-1$ в кольце $F_{1} \mathfrak{R}$ имеет место соотношение

$$
A(e, e) B^{n}(e) C(e) \neq \sum_{i, j} u_{i 1} h_{j} u_{i 2} .
$$

Это, как замечено вьше, равносильно тому, что для любого натурального числа $n>$ $m-1$ тождество (2) не вьполняется в многообразии $\mathfrak{X}_{m}$, что и требовалось доказать. Лемма 2 доказана.

Из лемм 1 и 2 очевидньм образом следует, что система тождеств, задающая многообразие $\mathfrak{X}$, не является независимой. Допустим тем не менее, что многообразие $\mathfrak{X}$ обладает независимьм базисом. Нетрудно убедиться в том, что если многообразие $\mathfrak{X}$ обладает 
независимым базисом тождеств, то многообразие $\mathfrak{X}$ обладает независимым базисом и как подмногообразие многообразия $\mathfrak{R}$. Зафиксируем некоторую независимую систему тождеств

$$
l_{1}(\vec{x})=0, \ldots, \quad l_{k}(\vec{x})=0, \ldots,
$$

порождающую многообразие $\mathfrak{X}$ как подмногообразие многообразия $\mathfrak{R}$. Поскольку система тождеств (49) порождает многообразие $\mathfrak{X}$, в многообразии

$$
\mathfrak{U}=\operatorname{var}\left\{l_{1}(\vec{x})=0, \ldots, l_{k}(\vec{x})=0, \ldots\right\} \cap \mathfrak{R}
$$

должны выполняться все тождества многообразия $\mathfrak{Y}$; в частности, должны выполняться тождество (1) и система тождеств вида (2), где $n \in \mathbb{N}$. Выполнимость тождества (1) и системы тождеств вида (2), где $n \in \mathbb{N}$, в многообразии $\mathfrak{U}$ равносильна вьполнимости этих тождеств в кольце счетного ранга, свободном в многообразии $\mathfrak{U}$. Последнее равносильно тому, что в кольце счетного ранга, свободном в многообразии $\mathfrak{U}$, выполняются равенства

$$
B\left(e_{1}, e\right) B(e) C(e) D(e)=B\left(e_{1}, e\right) C(e)
$$

и (6), где $n \in \mathbb{N}$, а $e$ и $e_{1}-$ свободные образующие кольца счетного ранга, свободного в многообразии $\mathfrak{U}$. Обозначим через $L$ множество многочленов $f(e)$ таких, что $f(e)=0$ - соотношение в кольце счетного ранга, свободном в многообразии $\mathfrak{U}$, полученное подстановкой конкретных элементов этого кольца вместо переменных в тождества системы (49). Пусть $J$ - идеал кольца $F \Re$, порожденный множеством $L$. Легко понять, что в кольце счетного ранга, свободном в многообразии $\mathfrak{U}$, равенства (6) и (50), где $n \in \mathbb{N}$, выполняются тогда и только тогда, когда многочлены (8) и

$$
B\left(e_{1}, e\right) B(e) C(e) D(e)-B\left(e_{1}, e\right) C(e)
$$

принадлежат идеалу $J$. Принадлежность этих многочленов идеалу $J$ равносильна тому, что в кольце $F \mathfrak{R}$ вьполняются равенства вида $(10)$ и

$$
B\left(e_{1}, e\right) B(e) C(e) D(e)-B\left(e_{1}, e\right) C(e)=\sum_{i, j} u_{i 1} h_{j} u_{i 2}
$$

где $h_{j} \in L$. Следует отметить, что равенств вида $(10)$ и (52) может выполняться в кольце $F \Re$, вообще говоря, несколько в зависимости от внешнего вида сумм (11). Однако мы зафиксируем вид этих равенств и в дальнейшем будем считать его неизменным. Заметим, что (52) - равенство многочленов (11) и (51). При этом в многочлене (11) может быть лишь конечное число одночленов. Поэтому сумма (11) содержит лишь конечное число элементов множества $L$. Следовательно, в вьводе тождества (1) задействовано лишь конечное число тождеств из системы (49). Пусть $r$ - число тождеств из системы (49), участвующих в вьводе тождества (1). В дальнейшем мы будем обозначать эти тождества $g_{1}(\vec{x})=0, \ldots, g_{r}(\vec{x})=0$. Оставшиеся тождества системы $(49)$ мы будем в дальнейшем обозначать $s_{1}(\vec{x})=0, \ldots, s_{k}(\vec{x})=0, \ldots$ Пусть, кроме того, $\mathbb{G} \rightleftharpoons\left\{g_{1}(\vec{x})=0, \ldots, g_{r}(\vec{x})=0\right\}, \mathbb{S} \rightleftharpoons\left\{s_{1}(\vec{x})=0, \ldots, s_{k}(\vec{x})=0, \ldots\right\}$. Заметим, что $\mathfrak{X}=\cap \mathfrak{X}_{m}$. Поэтому для любого тождества $s_{k}(\vec{x})=0$ из множества $\mathbb{S}$ существует натуральное число $m$ такое, что тождество $s_{k}(\vec{x})=0$ вьполняется в многообразии $\mathfrak{X}_{m}$. Из 
выполнимости тождества $s_{k}(\vec{x})=0$ в многообразии $\mathfrak{X}_{m}$ вытекает выполнимость этого тождества в кольце $F \mathfrak{X}_{m}$. Тождество $s_{k}(\vec{x})=0$ вьполняется в кольце $F \mathfrak{X}_{m}$ тогда и только тогда, когда в кольце $F \mathfrak{X}_{m}$ выполняется равенство $s_{k}(\vec{e})=0$. Обозначим через $S$ множество многочленов $f(\vec{e})$ таких, что $f(\vec{e})=0$ - соотношение в кольце $F \mathfrak{X}_{m}$, полученное подстановкой конкретных элементов кольца $F \mathfrak{X}_{m}$ вместо переменных в тождество (1) или в тождество (7). Пусть $I$ - идеал кольца $F \Re$, порожденный множеством $S$. Согласно лемме 1 имеет место равенство

$$
\mathfrak{X}_{m}=\operatorname{var}\left\{B(z, x) B(x) C(x) D(x)=B(z, x) C(x), A(x, x) B^{m-1}(x) C(x)=0\right\} .
$$

Следовательно, равенство $s_{k}(\vec{e})=0$ вьполняется в кольце $F \mathfrak{X}_{m}$ тогда и только тогда, ког да многочлен $s_{k}(\vec{e})$ принадлежит идеалу $I$. Принадлежность многочлена $s_{k}(\vec{e})$ идеалу $I$ равносильна тому, что в кольце $F \Re$ выполняется равенство вида

$$
s_{k}(\vec{e})=\sum_{i, j} u_{i 1} h_{j} u_{i 2}
$$

где $h_{j} \in S$. Отсюда непосредственно вытекает, что каждый одночлен многочлена $s_{k}(\vec{e})$ содержит одно из следуюших трех подслов:

$$
B(z, \vec{x}) B(\vec{x}) C(\vec{x}) D(\vec{x}), \quad B(z, \vec{x}) C(\vec{x}), \quad A(x, \vec{x}) B^{m-1}(\vec{x}) C(\vec{x}) .
$$

Аналогично тому, как мы из системы тождеств (49) выделили конечную подсистему $\mathbb{G}$, состоящую из тождеств, участвующих в выводе тождества (1), множество тождеств $\mathbb{S}$ можно представить в виде объединения бесконечного числа конечных подмножеств $\mathbb{S}_{i}, i \in \mathbb{N}$, таких, что для любого $i$ множество тождеств $\mathbb{S}_{i}$ - это пересечение множества тождеств $\mathbb{S}$ с множеством тождеств, используемых при вьводе тождества $A(x, x) B^{i}(x) C(x)=0$.

В силу леммы 2 для любого тождества $s(\vec{x})=0$ из множества $\mathbb{S}$ существует натуральное число $n_{s(\vec{x})=0}$ такое, что это тождество вьводимо из системы тождеств (1) и

$$
A(x, x) B^{n_{s_{1}(\vec{x})=0}}(x) C(x)=0,
$$

и невыводимо из тождества (1) и системы тождеств вида (3) для любого $m<n_{s}(\vec{x})=0$. Перенумеруем тождества из множества $\mathbb{S}$ таким образом, чтобы для любых $s(\vec{x})=0$, $t(\vec{x})=0 \in \mathbb{S}$ таких, что номер тождества $s(\vec{x})=0$ меньше номера тождества $t(\vec{x})=0$, имело место неравенство $n_{s(\vec{x})=0} \leqslant n_{t(\vec{x})=0}$. Предположим, что существуют тождество $s(\vec{x})=0$ из множества $\mathbb{S}$ и натуральное число $j \geqslant n_{s(\vec{x})=0}$ такие, что тождество $s(\vec{x})=0$ не принадлежит множеству $\mathbb{S}_{j}$. Поскольку $j \geqslant n_{s}(\vec{x})=0$, то в силу леммы 2 по определению числа $n_{s(\vec{x})=0}$ тождество $s(\vec{x})=0$ вьводимо из системы тождеств (1) и $A(x, x) B^{j}(x) C(x)=0$. Следовательно, тождество $s(\vec{x})=0$ выводимо из системы тождеств $\mathbb{S}_{j} \cup \mathbb{G}$. В силу того, что $s(\vec{x})=0 \notin \mathbb{S}_{j}$ и $s(\vec{x})=0 \notin \mathbb{G}$, имеет место соотношение $s(\vec{x})=0 \notin \mathbb{S}_{j} \cup \mathbb{G}$. Последнее, очевидно, противоречит независимости системы $\mathbb{S}$. Следовательно, мы убедились в том, что для любого тождества $s(\vec{x})=0$ из множества $\mathbb{S}$ это тождество принадлежит множеству $\mathbb{S}_{j}$ для любого натурального $j \geqslant n_{s(\vec{x})=0}$.

Пусть в кольце $F \Re$ вьполняется равенство

$$
A(e, e) B^{n_{s_{1}(\vec{x})=0}}(e) C(e)=\sum_{i, j} u_{i 1} h_{j} u_{i 2} .
$$


Представим сумму (11) в виде $T_{1}+T$, где $T_{1}$ и $T$ - многочлены вида (11), причем в $T_{1}$ для любого $j$ многочлен $h_{j}$ соответствует тождеству $s_{1}(\vec{x})=0$, а в $T$ ни один многочлен $h_{j}$ не соответствует тождеству $s_{1}(\vec{x})=0$. Заменив вхождения свободных образуюших в многочлене $T_{1}$ на переменные, получим некоторое тождество $T_{1}(\vec{x})=0$. Из равенства (54) и соотношения

$$
\sum_{i, j} u_{i 1} h_{j} u_{i 2}=T_{1}+T
$$

очевидным образом вытекает, что тождество $T_{1}(\vec{x})=0$ выполняется в многообразии $\mathfrak{X}$. Заменим в множестве $\mathbb{S}$ тождество $s_{1}(\vec{x})=0$ на тождество $T_{1}(\vec{x})=0$. Убедимся в том, что система тождеств

$$
\left((\mathbb{S} \cup \mathbb{G}) \backslash\left\{s_{1}(\vec{x})=0\right\}\right) \cup\left\{T_{1}(\vec{x})=0\right\}
$$

будет являться базисом многообразия $\mathfrak{X}$ и будет независима. Покажем сначала, что система тождеств (55) является базисом многообразия $\mathfrak{X}$. Для этого нужно убедиться в том, что все тождества многообразия $\mathfrak{X}$ вьводимы из множества тождеств (55). Пусть $t(\vec{x})=0$ - произвольное тождество, выполняющееся в многообразии $\mathfrak{X}$. Пусть $\mathbb{U}$ - множество тождеств, из которого вьводимо тождество $t(\vec{x})=0$. Очевидно, что $\mathbb{U} \subseteq \mathbb{S} \cup \mathbb{G}$. Предположим, что $\mathbb{U}$ - подмножество множества

$$
(\mathbb{S} \cup \mathbb{G}) \backslash\left\{s_{1}(\vec{x})=0\right\} .
$$

Тогда очевидно, что $\mathbb{U}$ - подмножество системы тождеств $(55)$ и тождество $t(\vec{x})=0$ выводимо из (55). Допустим теперь, что $\mathbb{U}$ не является подмножеством множества (56). Тогда тождество $s_{1}(\vec{x})=0$ сушественно участвует при выводе тождества $t(\vec{x})=0$ и принадлежит множеству $\mathbb{U}$. Однако, как замечено выше, тождество $s_{1}(\vec{x})=0$ выводимо из множества тождеств $(1),(53)$. Следовательно, множество $\mathbb{U}$ можно заменить на множество

$\left(\mathbb{U} \backslash\left\{s_{1}(\vec{x})=0\right\}\right) \cup\left\{B(z, x) B(x) C(x) D(x)=B(z, x) C(x), A(x, x) B^{n_{s_{1}(\vec{x})=0}}(x) C(x)=0\right\}$.

По определению множества $\mathbb{G}$ тождество (1) выводимо из множества $\mathbb{G}$, а по определению $T_{1}$ и $T$ в силу равенства

$$
A(e, e) B^{n_{s_{1}(\vec{x})=0}}(e) C(e)=\sum_{i, j} u_{i 1} h_{j} u_{i 2}=T_{1}+T
$$

тождество (53) выводимо из множества тождеств (55). Поэтому множество (57), а значит, и множество $\mathbb{U}$ можно заменить множеством (55). Отсюда в силу произвольности тождества $t(\vec{x})=0$ вытекает, что $(55)$ - базис многообразия $\mathfrak{X}$. Покажем теперь, что система тождеств $(55)$ независима. Пусть $t(\vec{x})=0$ - произвольное тождество, принадлежащее множеству (55) и выводимое из остальных тождеств множества (55). Предположим, что $t(\vec{x})=0$ - это $T_{1}(\vec{x})=0$. Тогда с учетом того, что тождество $s_{1}(\vec{x})=0$ выводимо из множества тождеств (55), получаем, что тождество $s_{1}(\vec{x})=0$ выводимо из множества тождеств (56). Это противоречит независимости системы тождеств $\mathbb{S} \cup \mathbb{G}$. Следовательно, тождество $t(\vec{x})=0$ отлично от $T_{1}(\vec{x})=0$. В этом случае с учетом того, 
что $T_{1}(\vec{x})=0$ выводимо из тождества $s_{1}(\vec{x})=0$, получаем, что тождество $t(\vec{x})=0$ выводимо из множества тождеств $(\mathbb{S} \cup \mathbb{G}) \backslash\{t(\vec{x})=0\}$. Это противоречит независимости системы тождеств $\mathbb{S} \cup \mathbb{G}$. Следовательно, система тождеств (55) действительно независима. Заметим, что, действуя совершенно аналогично случаю тождества $s_{1}(\vec{x})=0$, мы можем заменить тождества $s_{k}(\vec{x})=0$ на тождества $T_{k}(\vec{x})=0$ для любого натурального числа $k$. В силу того, что в левой части равенства

$$
A(e, e) B^{n_{s_{k}}(\vec{x})=0}(e) C(e)=\sum_{i, j} u_{i 1} h_{j} u_{i 2}
$$

стоит многочлен, зависящий только от образующего $е$, без ограничения общности можно считать, что все одночлены суммы (11) тоже зависят только от образующего $е$. Отсюда непосредственно вытекает, что в многочленах $T_{k}$ и $T$, где $T_{k}$ и $T$ - многочлены вида $(11)$, причем в $T_{k}$ для любого $j$ многочлен $h_{j}$ соответствует тождеству $s_{k}(\vec{x})=0$, а в $T$ ни один многочлен $h_{j}$ не соответствует тождеству $s_{k}(\vec{x})=0$, все одночлены зависят тоже только от образующего $e$. Поэтому тождество $T_{k}(\vec{x})=0$ будет тождеством одной переменной, и мы можем писать просто $T_{k}(x)=0$. Таким образом, в многообразии $\mathfrak{X}$ имеется независимый базис вида $\mathbb{T} \cup \mathbb{G}$, где $\mathbb{T}-$ множество тождеств, полученное из множества тождеств $\mathbb{S}$ заменой каждого тождества $s_{k}(\vec{x})=0$ на $T_{k}(x)=0$. Если мы вместо равенства (58) рассмотрим равенство

$$
A(e, e) B^{r}(e) C(e)=\sum_{i, j} u_{i 1} h_{j} u_{i 2}
$$

где $r>n_{s_{k}(\vec{x})=0}$, то вместо $T_{k}(x)=0$ получим некоторое тождество $T_{k, r}(x)=0$. Действуя так же, как и в случае тождества $T_{k}(x)=0$, можно показать, что система тождеств $\left((\mathbb{T} \cup \mathbb{G}) \backslash\left\{T_{k}(x)=0\right\}\right) \cup\left\{T_{k}, r(x)=0\right\}$ независима и является базисом многообразия $\mathfrak{X}$.

Рассмотрим равенства (58) и (59), где $r>n_{s_{k}(\vec{x})=0}$. В силу сказанного вьше эти равенства можно представить в виде

$$
\begin{gathered}
A(e, e) B^{r}(e) C(e)=T_{k, r}(e)+T(e), \\
A(e, e) B^{n_{s_{k}}(\vec{x})=0}(e) C(e)=T_{k}(e)+T^{\prime}(e),
\end{gathered}
$$

где $T(e)$ и $T^{\prime}(e)$ - многочлены вида (11) такие, что для любого $j$ многочлен $h_{j}$ не соответствует тождеству $T_{k}(x)=0$. Заметим, что в силу леммы 2 имеет место равенство

$$
A(e, e) B^{n_{s_{k}}(\vec{x})=0}(e) C(e)=A(e, e) B^{r}(e) C(e) D^{r-n_{s_{k}}(\vec{x})=0}+\sum_{i, j} u_{i 1} h_{j} u_{i 2},
$$

где (11) - многочлен такой, что для любого $j$ многочлен $h_{j}$ получен из тождества $(1)$. По определению множества $\mathbb{G}$ одночлены многочлена (11) можно перегруппировать таким образом, чтобы он попрежнему имел вид (11) и, кроме того, для любого $j$ многочлен $h_{j}$ был получен из одного из тождеств, принадлежащих множеству $\mathbb{G}$. Отсюда в силу равенства (60) получаем равенство

$$
\begin{aligned}
A(e, e) B^{n_{s_{k}}(\vec{x})=0}(e) C(e) & =A(e, e) B^{r}(e) C(e) D^{r-n_{s_{k}}(\vec{x})=0}+\sum_{i, j} u_{i 1} h_{j} u_{i 2} \\
& =\left(T_{k, r}(e)+T(e)\right) D^{r-n_{s_{k}}(\vec{x})=0}+\sum_{i, j} u_{i 1} h_{j} u_{i 2} .
\end{aligned}
$$


Следовательно, в силу равенства (61) имеет место соотношение

$$
T_{k}(e)=T_{k, r}(e) D^{r-n_{s_{k}}(\vec{x})=0}+T^{\prime \prime}(e),
$$

где $T^{\prime \prime}(e)$ - многочлен вида (11) такой, что для любого $j$ многочлен $h_{j}$ получен из тождества (1). Поскольку равенство вида (62) имеет место для любого $r>n_{s_{k}(\vec{x})=0}$, тождество $T_{k}(x)=0$ можно заменить в базисе $\mathbb{T} \cup \mathbb{G}$ тождеством вида

$$
\sum_{j} B\left(z_{j}(x), x\right) B^{q_{j}}(x) C(x)=0
$$

для подходящих слов $z_{j}(x)$ и сколь угодно больших натуральных чисел $q_{j}$. Используя рассуждения, аналогичные рассуждениям, использованным при доказательстве леммы 4 , можно показать, что для любого $k$ тождество $T_{k}(x)=0$ можно заменить в базисе $\mathbb{T} \cup \mathbb{G}$ тождеством вида

$$
\sum_{j} A(x, x) B^{q_{j}}(x) C(x)=0,
$$

которое мы будем в дальнейшем обозначать

$$
P_{k, q_{1}, \ldots, q_{j_{k}}}(x)=0
$$

При этом (63) будет удовлетворять тем же условиям, что и тождество $T_{k}(x)=0$. Следовательно, система тождеств $\mathbb{P} \cup \mathbb{G}$, где $\mathbb{P} \rightleftharpoons\left\{P_{k}, q_{1}, \ldots, q_{j_{k}}(x)=0 \mid k \in \mathbb{N}\right\}$, будет независимым базисом многообразия $\mathfrak{X}$. Заметим, что замену тождеств вида $T_{k}(x)=0$ в базисе многообразия $\mathfrak{X}$ на тождества вида (63) мы можем осуществлять последовательно, т.е. при замене тождества $T_{k}(x)=0$ на (63) тождества

$$
P_{l, q_{1}, \ldots, q_{j_{l}}}(x)=0
$$

где $l<k$, можно считать уже известными. Поскольку, как замечено выше, числа $q_{1}, \ldots$, $q_{j_{l}}$ можно брать сколь угодно большими, мы можем полагать, что для любых двух тождеств (63) и (64) из множества $\mathbb{P}$ если $l<k$, то выполняется соотношение

$$
\max \left\{q_{l 1}, \ldots, q_{l j_{l}}\right\}<\min \left\{q_{k 1}, \ldots, q_{k j_{k}}\right\}
$$

Обозначим через $\mathbb{P}_{1}$ конечное подмножество множества $\mathbb{P}$, состоящее из всех тех тождеств множества $\mathbb{P}$, которые используются при вьводе тождества

$$
A(x, x) B(x) C(x)=0 .
$$

Пусть

$$
P_{1, q_{1}, \ldots, q_{j_{1}}}(x)=0
$$

- тождество с наименьшим номером, принадлежашее множеству $\mathbb{P}$, но не принадлежащее множеству $\mathbb{P}_{1}$. Покажем, что оно вьводимо из множества тождеств

$$
(\mathbb{P} \cup \mathbb{G}) \backslash\left\{P_{1, q_{1}, \ldots, q_{j_{1}}}(x)=0\right\} .
$$

Для этого нам потребуется предварительная 
Лемма 5. Пусть (63) и (64) - два тождества такие, что для некоторого натурального числа $г$ выполняются следующие условия:

$$
j_{l}=j_{k}, \quad q_{l 1}+r=q_{k 1}, \quad \ldots, \quad q_{l j_{l}}+r=q_{k j_{k}} .
$$

Тогда тохсдество (64) выводимо из тохсдеств (1) и (63).

ДокАЗАТЕЛЬСТво проведем индукцией по числу $d=q_{k 1}+\cdots+q_{k j_{k}}$. Допустим, что $d=1$. Тогда утверждение очевидно. Предположим, что лемма вьполняется для некоторого числа $d$ и покажем, что она справедлива и для числа $d+1$. Пусть (63) и $(64)-$ тождества, удовлетворяюшие соотношениям (68) для некоторого натурального числа $r$, и $d+1=q_{k 1}+\cdots+q_{k j_{k}}$. Покажем, что из тождеств (1) и (63) следует тождество

$$
P_{k, q_{k 1}-1, q_{k 2}-1, \ldots, q_{k j_{k}}-1}(x)=0 \text {. }
$$

В самом деле,

$$
\begin{aligned}
P_{k, q_{k 1}, q_{k 2}, \ldots, q_{k j_{k}}}(x)= & A(x, x) B^{q_{k 1}}(x) C(x)+A(x, x) B^{q_{k 2}}(x) C(x)+\cdots \\
& +A(x, x) B^{q_{k j_{k}}-1}(x) C(x)=0 .
\end{aligned}
$$

Следовательно, из тождества (63) вытекает тождество

$$
\begin{aligned}
& A(x, x) B^{q_{k 1}}(x) C(x) D(x)+A(x, x) B^{q_{k 2}}(x) C(x) D(x)+\cdots \\
& \quad+A(x, x) B^{q_{k j_{k}}-1}(x) C(x) D(x)=0 .
\end{aligned}
$$

Применив к каждому одночлену этого тождества тождество (1), получим тождество

$$
\begin{aligned}
& A(x, x) B^{q_{k 1}-1}(x) C(x)+A(x, x) B^{q_{k 2}-1}(x) C(x)+\cdots+A(x, x) B^{q_{k j_{k}}-1}(x) C(x) \\
& \quad=P_{k, q_{k 1}-1, q_{k 2}-1, \ldots, q_{k j_{k}}-1}(x)=0 .
\end{aligned}
$$

Тождество (64) выводимо из тождеств (1) и (69) по предположению индукции, поэтому в силу того, что тождество (69) выводимо из тождеств (1) и (63), получаем выводимость тождества (64) из тождеств (1) и (63). Лемма 5 доказана.

Доказательства вьводимости тождества (66) из множества тождеств (67) проведем индукцией по степени старшего члена многочлена $P_{1, q_{1}, \ldots, q_{j_{1}}}(x)$. Поскольку база индукции очевидна, для доказательства требуемого факта нам достаточно показать, что в многообразии, заданном системой тождеств (67), имеет место тождество

$$
P_{1, q_{1}, \ldots, q_{j_{1}}}(x)=\sum_{l} A(x, x) B^{a_{l}}(x) C(x),
$$

где $\max _{l}\left\{a_{l}\right\}<\max \left\{q_{1}, \ldots, q_{j_{1}}\right\}$. Пусть

$$
P_{2, q_{21}, \ldots, q_{2 j_{2}}}(x)=0
$$

- тождество, отличное от тождества (66), принадлежащее множеству $\mathbb{P}$, но не принадлежащее множеству $\mathbb{P}_{1}$. Без ограничения общности в силу тождества $2 x=0$ можно 
считать, что числа $q_{11}, \ldots, q_{1 j_{1}}, q_{21}, \ldots, q_{2 j_{2}}$ попарно различны и удовлетворяют следуюшим неравенствам: $q_{11}<\cdots<q_{1 j_{1}}, q_{21}<\cdots<q_{2 j_{2}}$. Кроме того, поскольку (66) тождество с наименьшим номером, принадлежащее множеству $\mathbb{P}$, но не принадлежашее множеству $\mathbb{P}_{1}$, имеет место неравенство $q_{1 j_{1}}<q_{21}$. Пусть $r=q_{2 j_{2}}-q_{1 j_{1}}$ и $r<q_{21}$. Тогда из тождества (70) в силу леммы 5 следует тождество

$$
P_{2, q_{21}-r, \ldots, q_{2 j_{2}}-r}(x)=0,
$$

и в качестве $\sum_{l} A(x, x) B^{a_{l}}(x) C(x)$ мы можем взять многочлен

$$
P_{1, q_{1}, \ldots, q_{j_{1}}}(x)+P_{1, q_{21}-r, \ldots, q_{2 j_{2}}-r}(x) .
$$

Предположим, что неравенство $r<q_{21}$ не вьполняется, и покажем, что в этом случае тождество (70) можно заменить другим тождеством, которое выводимо из системы тождеств (67) и для которого неравенство $r<q_{21}$ справедливо. Если имеет место неравенство $r \geqslant q_{21}$, то вместо тождества (71) можно рассмотреть тождество

$$
P_{2,1, q_{21}-q_{11}+1, \ldots, q_{2 j_{2}}-q_{11}+1}(x)=0 .
$$

По определению множества $\mathbb{P}_{1}$ мы можем из множества тождеств $\mathbb{P}_{1} \cup \mathbb{G}$ вьвести тождество (65). Складьвая тождества (65) и (72), получим тождество

$$
P_{2, q_{21}-q_{11}+1, \ldots, q_{2 j_{2}}-q_{11}+1}(x)=0 .
$$

Теперь в качестве числа $r$ мы можем взять число $q_{2 j_{2}}-q_{11}+1-q_{1 j_{1}}$ и снова проверить, выполняется ли необходимое неравенство. Поскольку на каждом шаге число $r$ становится меньше, этот процесс оборвется на конечном шаге и мы либо получим требуемое неравенство, либо сведем многочлен $P_{2}, q_{21}, \ldots, q_{2 j_{2}}(x)$ к одночлену и сможем воспользоваться леммой 1. Итак, мы убедились, что тождество (66) выводимо из множества тождеств (67). Это противоречит независимости системы тождеств $\mathbb{P} \cup \mathbb{G}$, а значит, и системы тождеств $\mathbb{S} \cup \mathbb{G}$. Полученное противоречие доказьвает, что многообразие $\mathfrak{X}$ не обладает независимым базисом тождеств. Теорема доказана.

В работе [10] замечено, что многообразие, не имеющее независимого базиса тождеств, построенное в [4], является пересечением независимо базируемого и конечно базируемого многообразий. Там же отмечен вопрос М. В. Сапира: каждое ли многообразие полугрупп является пересечением конечного числа независимо базируемых многообразий? В свете этого вопроса представляет интерес следующее

ПРЕДЛОЖЕНИЕ. Многообразие колеи, $\mathfrak{X}$ является пересечением независимо базируемого и конечно базируемого многообразий.

ДокАЗАТЕЛЬСтво. Для доказательства нам нужно показать, что сушествует конечно базируемое многообразие $\mathfrak{Y}_{1}$ и независимо базируемое многообразие $\mathfrak{Y}_{2}$ такие, что их пересечение является многообразием $\mathfrak{X}$. Обозначим через $\mathfrak{Y}_{1}$ многообразие колец, заданное тождеством (1), а через $\mathfrak{Y}_{2}$ - многообразие колец, заданное системой тождеств вида $(2)$, где $n \in \mathbb{N}$, и тождествами $2 x=0,\left(x_{1} x_{2}\right)\left(x_{3} x_{4}\right)=0$. Очевидно, что многообразие $\mathfrak{Y}_{1}$ конечно базируемо и что пересечение многообразий $\mathfrak{Y}_{1}$ и $\mathfrak{Y}_{2}$ равно многообразию $\mathfrak{X}$. Следовательно, для доказательства предложения нам достаточно показать, 
что многообразие $\mathfrak{Y}_{2}$ обладает независимым базисом тождеств. Допустим противное. Тогда либо существует натуральное число $k$ такое, что тождество

$$
A(x, x) B^{k}(x) C(x)=0
$$

выводимо из множества тождеств

$$
\left\{A(x, x) B^{n}(x) C(x)=0,2 x=0,\left(x_{1} x_{2}\right)\left(x_{3} x_{4}\right)=0 \mid n \in \mathbb{N} \backslash\{k\}\right\},
$$

либо тождество $2 x=0$ выводимо из множества тождеств

$$
\left\{A(x, x) B^{n}(x) C(x)=0,\left(x_{1} x_{2}\right)\left(x_{3} x_{4}\right)=0 \mid n \in \mathbb{N}\right\}
$$

либо тождество $\left(x_{1} x_{2}\right)\left(x_{3} x_{4}\right)=0$ вьводимо из множества тождеств

$$
\left\{A(x, x) B^{n}(x) C(x)=0,2 x=0 \mid n \in \mathbb{N}\right\} .
$$

Предположим сначала, что тождество $2 x=0$ выводимо из множества тождеств (75). Пусть $\mathfrak{Y}_{2}^{\prime}$ - многообразие колец, заданное системой тождеств $(75), F \mathfrak{Y}_{2}^{\prime}$ - кольцо счетного ранга, свободное в многообразии $\mathfrak{Y}_{2}^{\prime}, F$ - кольцо счетного ранга, свободное в многообразии всех колец. Как и раньше, образуюшие свободных колец будем обозначать элементами множества

$$
\left\{e, e_{1}, \ldots, e_{n}, \ldots\right\} \text {. }
$$

Заметим, что тождество $2 x=0$ выводимо из множества тождеств (75) тогда и только тогда, когда оно выполняется в многообразии $\mathfrak{Y}_{2}^{\prime}$. Выполнимость тождества $2 x=0$ в многообразии $\mathfrak{Y}_{2}^{\prime}$ равносильна выполнимости этого тождества в кольце $F \mathfrak{Y}_{2}^{\prime}$. Тождество $2 x=0$ истинно в кольце $F \mathfrak{Y}_{2}^{\prime}$ тогда и только тогда, когда в этом кольце вьполняется равенство $2 e=0$. Истинность равенства $2 e=0$ в кольце $F \mathfrak{Y}_{2}^{\prime}$ равносильна тому, что в кольце $F$ выполняется равенство вида

$$
2 e=\sum_{i, j} u_{i 1} h_{j} u_{i 2},
$$

где для любого натурального числа $j$ многочлен $h_{j}$ получен из одного из многочленов $A(x, x) B^{n}(x) C(x),\left(x_{1} x_{2}\right)\left(x_{3} x_{4}\right)$, где $n \in \mathbb{N}$, подстановкой конкретньг элементов кольца $F$ вместо переменных. По определению многочленов $h_{j}$ для любого $j$ степени одночленов, входящих в $h_{j}$, не меньше 4 . Следовательно, ни один одночлен многочлена (11) не имеет степени меньше 4. Отсюда в силу свободы кольца $F$ непосредственно вытекает, что равенство

$$
2 e=\sum_{i, j} u_{i 1} h_{j} u_{i 2}
$$

ложно в кольце $F$. Поэтому в силу сказанного выше тождество $2 x=0$ не вьводимо из множества тождеств (75).

Допустим теперь, что тождество $\left(x_{1} x_{2}\right)\left(x_{3} x_{4}\right)=0$ вьводимо из множества тождеств (76). Пусть $\mathfrak{Y}_{2}^{\prime \prime}-$ многообразие колец, заданное системой тождеств $(76), F \mathfrak{Y}_{2}^{\prime \prime}-$ кольцо счетного ранга, свободное в многообразии $\mathfrak{Y}_{2}^{\prime \prime}, F^{\prime}$ - кольцо счетного ранга, свободное в многообразии всех колец характеристики 2. Как и раньше, образующие свободных колец будем обозначать элементами множества (77). 
Заметим, что тождество $\left(x_{1} x_{2}\right)\left(x_{3} x_{4}\right)=0$ вьводимо из множества тождеств (76) тогда и только тогда, когда оно вьполняется в многообразии $\mathfrak{Y}_{2}^{\prime \prime}$. Вьполнимость тождества $\left(x_{1} x_{2}\right)\left(x_{3} x_{4}\right)=0$ в многообразии $\mathfrak{Y}_{2}^{\prime \prime}$ равносильна вьполнимости этого тождества в кольце $F \mathfrak{Y}_{2}^{\prime \prime}$. Тождество $\left(x_{1} x_{2}\right)\left(x_{3} x_{4}\right)=0$ истинно в кольце $F \mathfrak{Y}_{2}^{\prime \prime}$ тогда и только тогда, когда в этом кольце выполняется равенство $\left(e_{1} e_{2}\right)\left(e_{3} e_{4}\right)=0$. Истинность равенства $\left(e_{1} e_{2}\right)\left(e_{3} e_{4}\right)=0$ в кольце $F \mathfrak{Y}_{2}^{\prime \prime}$ равносильна тому, что в кольце $F^{\prime}$ вьполняется равенство вида

$$
\left(e_{1} e_{2}\right)\left(e_{3} e_{4}\right)=\sum_{i, j} u_{i 1} h_{j} u_{i 2},
$$

где для любого натурального числа $j$ многочлен $h_{j}$ получен из одного из многочленов $A(x, x) B^{n}(x) C(x)$, где $n \in \mathbb{N}$, подстановкой конкретных элементов кольца $F^{\prime}$ вместо переменных. По определению многочленов $h_{j}$ для любого $j$ степени одночленов, входящих в $h_{j}$, не меньше 24 . Следовательно, ни один одночлен многочлена (11) не имеет степени меньше 24. Отсюда в силу свободы кольца $F^{\prime}$ непосредственно вытекает, что равенство

$$
\left(e_{1} e_{2}\right)\left(e_{3} e_{4}\right)=\sum_{i, j} u_{i 1} h_{j} u_{i 2}
$$

ложно в кольце $F^{\prime}$. Поэтому в силу сказанного выше тождество $\left(x_{1} x_{2}\right)\left(x_{3} x_{4}\right)=0$ не выводимо из множества тождеств (76).

Итак, для завершения доказательства предложения нам осталось рассмотреть случай, когда тождество (73) выводимо из множества тождеств (74).

Допустим, что тождество (73) выводимо из множества тождеств (74). Пусть $\mathfrak{Y}_{2}^{\prime \prime \prime}-$ многообразие колец, заданное системой тождеств $(74)$. Обозначим через $F \mathfrak{Y}_{2}^{\prime \prime \prime}$ кольцо счетного ранга, свободное в многообразии $\mathfrak{Y}_{2}^{\prime \prime \prime}, F^{\prime \prime}$ - кольцо счетного ранга, свободное в многообразии всех метабелевых колец характеристики 2. Как и раньше, образуюшие свободных колец будем обозначать элементами множества (77).

Тождество (73) выводимо из множества тождеств (74) тогда и только тогда, когда оно вьполняется в многообразии $\mathfrak{Y}_{2}^{\prime \prime \prime}$. Выполнимость тождества (73) в многообразии $\mathfrak{Y}_{2}^{\prime \prime \prime}$ равносильна вьполнимости этого тождества в кольце $F \mathfrak{Y}_{2}^{\prime \prime \prime}$. Тождество $(73)$ истинно в кольце $F \mathfrak{Y}_{2}^{\prime \prime \prime}$ тогда и только тогда, когда в этом кольце вьполняется равенство $A(e, e) B^{k}(e) C(e)=0$. Истинность равенства $A(e, e) B^{k}(e) C(e)=0$ в кольце $F \mathfrak{Y}_{2}^{\prime \prime \prime}$ равносильна тому, что в кольце $F^{\prime \prime}$ вьполняется равенство вида

$$
A(e, e) B^{k}(e) C(e)=\sum_{i, j} u_{i 1} h_{j} u_{i 2},
$$

где для любого натурального числа $j$ многочлен $h_{j}$ получен из одного из многочленов $A(x, x) B^{n}(x) C(x)$, где $n \in \mathbb{N} \backslash\{k\}$, подстановкой конкретных элементов кольца $F^{\prime \prime}$ вместо переменных. Поскольку в одночлен $A(e, e) B^{k}(e) C(e)$ входит только образующий $e$, без ограничения общности в силу свободы кольца $F^{\prime \prime}$ можно считать, что многочлен (11) тоже зависит только от $e$. В силу дистрибутивности можно считать, что все многочлены, входяшие в произведение (9), кроме разве лишь $h_{j}$, являются одночленами. Пусть $W \rightleftharpoons\left\{A(e, e) B^{n}(e) C(e) \mid n \in \mathbb{N}\right\}$.

Лемма 6. Сумма (11) представима в виде $f_{1}+f_{2}$, где $f_{1}$ - многочлен, который сам может быть представлен в виде (11), каждый одночлен многочлена $f_{1}$ принадлежит множеству $W$, а $f_{2}$ - многочлен, ни один одночлен которого не принадлежит множеству $W$. 
ДокАЗАТЕЛЬСтво.Доказательство проведем индукцией по числу одночленов в сумме (11). Допустим, что число $d$ одночленов в сумме (11) равно нулю. Тогда утверждение очевидно. Допустим, что утверждение справедливо для некоторого числа одночленов $d$. Покажем, что оно верно и для $d+1$. Пусть число одночленов в сумме $(11)$ не больше $d+1$. Тогда выполняется равенство (12). Так как число одночленов в сумме (13) не превосходит $d$, то по предположению индукции имеет место равенство (14). Рассмотрим многочлен (15). В силу метабелевости кольца $F^{\prime \prime}$ получаем, что либо $h_{1}=0$, либо $h_{1}=A(e, e) B^{m}(e) C(e)$, либо

$$
h_{1}=A(e, e) B^{m}(e) C(e)+A(y, e) B^{m}(e) C(e)+A(e, y) B^{m}(e) C(e),
$$

где $y$ - многочлен, отличньй от нуля и не содержащий одночлена $e$. Поскольку многочлен $y$ входит в многочлены (16) и (17) линейно, в силу дистрибутивности $y$ можно считать одночленом. При доказательстве леммы 3 мы показали, что имеют место соотношения (18) и (19). Поэтому требуемым представлением будет представление (23) или (24). Лемма 6 доказана.

Завершить доказательство предложения теперь можно несложными рассуждениями по схеме доказательства леммы 4.

Существование многообразий ассоциативных и лиевых колец, не имеющих конечного базиса тождеств, а также результаты, полученные в статье, делают естественными следуюшие два вопроса.

Вопрос 1. Существует ли многообразие ассоциативных (лиевых) колец, не обладающее независимьм базисом?

Вопрос 2. Каждое ли многообразие колец является пересечением конечного числа независимо базируемых многообразий?

\section{СПИСОК ЦИТИРОВАННОЙ ЛИТЕРАТУРЫ}

[1] Бахтурин Ю. А. Тождества в алгебрах Ли. М.: Наука, 1985.

[2] Айзенштат А.Я. О покрытиях в решетке многообразий полугрупп // Современый анализ и геометрия. Ленинград, 1972. С. 3-11.

[3] Мартынова Т. А. Группоид 0-приведенных многообразий полугрупп // Исследования по современной алгебре. Свердловск, 1979. С. 96-115.

[4] Трахтман А. Н. Многообразие полугрупп без неприводимого базиса тождеств // Матем. заметки. 1977. Т. 21. №6. С. 865-872.

[5] Pollak G. Some lattices of varieties containing elements without cover // Noncommutative Structures in Algebra and Geometric Combinatorics. Rome, 1981. P. 91-96.

[6] Сапир М.В. Малые, кроссовы и предельные многообразия полугрупп // 16 Всесоюзная алгебраическая конференция. Тезисы докладов. Ч. 2. Ленинград, 1981. С. 142-143.

[7] Трахтман А.Н. Шестиэлементная полугруппа, порождающая многообразие с континуумом подмногообразий // Алгебраические системы и их многообразия. Свердловск, 1988. C. $138-143$.

[8] Клейман Е.И. Об условии покрытия в решетке многообразий инверсных полугрупп // Исследования алгебраических систем по свойствам их подсистем. Свердловск, 1980. С. 76-91.

[9] Клейман Ю. Г. О некоторых вопросах теории многообразий групп // Изв. АН СССР. Сер. матем. 1983. Т. 47. №1. С. 37-74.

[10] Шеврин Л.Н., Волков М.В. Тождества полугрупп // Изв. вузов. Матем. 1985. № 11. C. 3-47.

Уральский государственный университет, г. Екатеринбург

Поступило

17.02.1999

Исправленный вариант

14.12 .2000 